\title{
El control gubernamental de RTVE y el pluralismo en los informativos
}

\author{
Xosé Soengas Pérez \\ Universidad de Santiago de Compostela \\ jose.soengas@usc.es \\ Ana Isabel RodRÍGUEZ VÁZQUEZ \\ Universidad de Santiago de Compostela \\ anaisabel.rodriguez.vazquez@usc.es
}

Recibido: 15 de mayo de 2014

Aceptado: 10 de noviembre de 2014

\section{Resumen}

El objetivo de esta investigación es analizar y comparar los telediarios de etapas diferentes para conocer las características de la política informativa de RTVE durante el último mandato del PSOE y del PP, y ver si los cambios de Gobierno y en la dirección de informativos han influido en la línea editorial de la televisión pública. El grado de pluralismo formal registrado no se corresponde siempre con un enfoque neutral y con un tratamiento riguroso de las noticias. Los datos reflejan que en muchos casos no existe una correspondencia entre la dimensión cuantitativa y cualitativa del relato. Los resultados de la investigación confirman que el consenso político sobre la televisión pública favorece el rigor, la transparencia y el pluralismo informativo, y disminuye la manipulación y la censura.

Palabras clave: televisión, información, manipulación, censura.

\section{Governmental Control of Spanish Public Televison and Pluralism in Newcasts}

\begin{abstract}
The goal of this study is to analyze and compare television newscasts broadcast during the most recent terms of office for Spain's two primary political parties: the PSOE (the Socialist Party) and the PP (the conservative People's Party); in order to better understand the characteristics of RTVE's (The Spanish Radio and Television Corporation, a state-owned entity) communications strategy and to determine if changes in the governing party as well as at the senior management level in the newsroom have influenced the editorial policy of public television. The level of formal pluralism recorded does not always imply a neutral approach to reporting, nor does it mean that rigorous standards are always enforced. The facts show that in many cases there is no relationship between the quantitative and qualitative elements of any given story. The results of this study confirm that political consensus with regards to public television improves accuracy, transparency and pluralism of information, in addition to decreasing manipulation and censorship.
\end{abstract}

Keywords: Television, information, manipulation, censorship.

\section{Referencia normalizada}

SOENGAS PÉREZ, Xosé y RODRÍGUEZ VÁZQUEZ, Ana Isabel (2015): "El control gubernamental de RTVE y el pluralismo en los informativos". Estudios sobre el Mensaje Periodístico. Vol. 21, Núm. 2 (julio-diciembre), págs.: 1225-1240. Madrid, Servicio de Publicaciones de la Universidad Complutense.

Sumario: 1. Introducción y marco teórico; 1.1. RTVE debe ser un servicio público; 1.2. Una televisión pública basada en un modelo comercial y competitivo. 2. Objeto de estudio. 3. Objetivos e hipótesis. 4. Metodología. 5. Análisis de los datos y resultados; 5.1. El pluralismo formal en las noticias de TVE; 5.2. El pluralismo formal en la información política; 5.3. Los predominios temáticos de cada etapa; 5.4. La forma y el fondo: la correspondencia entre el tratamiento formal y el enfoque de las noticias; 5.5 . La línea editorial de TVE. 6. Conclusiones. 7. Referencias bibliográficas. 


\section{Introducción y marco teórico}

La estructura de RTVE dispone de los recursos y de los mecanismos necesarios para garantizar la independencia de sus servicios informativos. Pero los mismos elementos que permiten mantener la calidad y el pluralismo pueden convertirse en aliados de la censura, de la manipulación y del control inadecuado de los contenidos (Soler Campillo, 2005: web).

\subsection{RTVE debe ser un servicio público}

Los ciudadanos tienen derecho al acceso libre y gratuito a una información veraz, rigurosa y plural, y los medios de comunicación públicos tienen la obligación y la responsabilidad de satisfacer esa necesidad (Palacio, 2001). RTVE debe estar despolitizada, al servicio de la sociedad, especialmente los informativos, porque si está controlada por el poder político se convierte en una televisión de partido o en una televisión gubernamental que no cumple la función social que le corresponde (Pérez Ornia, 1989). Para que exista calidad, pluralidad real y diversidad en los contenidos de los telediarios, RTVE tiene que aplicar una política informativa coherente, que garantice unas condiciones que permitan a los periodistas desarrollar su trabajo con criterios profesionales, con rigor y con independencia (Soler Campillo, 2005: web).

En Europa existen varios modelos de televisión pública (Campos Freire, 2013: web), pero en España nunca ha habido un acuerdo sólido sobre la financiación (De Mateo y Bergés, 2008), ni sobre la fórmula para nombrar el presidente de RTVE, y por eso el modelo implantado nunca ha tenido el consenso suficiente para fijar unas directrices que satisfagan las diferentes sensibilidades políticas y sociales (Palacio, 2012). RTVE tiene que estar blindada para evitar injerencias que conlleven usos partidistas y una politización de la cadena (Humanes et al., 2013: web). El nombramiento del presidente es uno de los aspectos más polémico y cuestionado por muchos expertos, porque consideran que la fórmula actual perjudica la calidad, el pluralismo de los contenidos y la neutralidad de la información, y no garantiza la independencia profesional de los redactores (Bustamante, 2013). La dependencia gubernamental de RTVE se acentúa en periodos de mayoría absoluta y es un obstáculo para que en los telediarios exista un pluralismo real. Los diferentes gobiernos se han asegurado el control de la televisión pública como estrategia política y ha predominado el continuismo ideológico sobre una renovación profunda de la cadena (Castillo Esparcia, 2012: web).

\subsection{Una televisión pública basada en un modelo comercial y competitivo}

La televisión pública en Europa ha perdido peso en la última década y RTVE no es una excepción. La lucha por las audiencias desvirtúa su identidad y la aproxima cada vez más al modelo de las privadas porque, al situarse en un escenario comercial, tiene que competir con otros esquemas de producción (Pestano Rodríguez, 2008: web). Además, la aparición de nuevas formas de distribución y de consumo de la información obliga a los servicios informativos a realizar una serie de esfuerzos para los que se necesitan recursos, lo cual crea unas dependencias económicas permanentes y a veces difíciles de asumir (Campos Freire, 2013: web). RTVE está inmersa en un sistema competitivo que contradice y dificulta la prestación de un servicio alejado de intereses económi- 
cos y políticos y de resultados inmediatos de audiencia. Se trata de un servicio público que adopta los valores y las normas de un modelo comercial y la audiencia se convierte en el factor determinante de la política informativa y de las estrategias de programación para competir con las demás televisiones (Bustamante, 2013).

El contexto social condiciona el contexto informativo y los telediarios son los más afectados por las exigencias de un escenario que no admite los procesos sosegados de búsqueda de datos que requieren algunas informaciones, ni presta atención a los intereses y a las necesidades de las minorías porque no generan altos índices de audiencia (Díaz Arias, 2008: web). RTVE tiene que respetar la diversidad y dar un servicio adecuado a todos los sectores de la sociedad (Humanes, 2001: web), y cuando se compite con otros canales que se basan en modelos comerciales, la selección de los contenidos está determinada por unos objetivos de rentabilidad económica, que en la mayoría de los casos no responden a un servicio público, sino a una lucha por estar a la altura de la competencia o por superarla (Ortells Badenes, 2009: web).

Los informativos de RTVE se rigen por criterios muy similares a los de las cadenas privadas (Retis et al., 2010). La audiencia es la referencia principal que sirve para medir el éxito de los telediarios, en lugar de guiarse exclusivamente por parámetros de calidad y de servicio público (Díaz Arias, 2006). La búsqueda de unos resultados numéricos influye directamente en la selección temática, en el enfoque y en el tratamiento de los contenidos (León, 2010). El espectáculo informativo y el traslado a las noticias de fórmulas narrativas propias del magazín, de los espacios de entretenimiento y de los programas de ficción son recursos habituales para conseguir audiencia (García Avilés, 2007: web).

\section{Objeto de estudio}

El objeto de estudio de esta investigación se centra en el análisis de las noticias referidas a España emitidas por TVE durante 2008, 2012 y 2013, tres años que consideramos adecuados para comprobar si existen distintas formas de dirigir los informativos de la televisión pública. Los telediarios de 2008 coinciden con un momento del Gobierno del PSOE en el que RTVE estaba dirigida por un presidente cuyo nombramiento había sido consensuado entre los principales partidos; los de 2012 corresponden al primer año del Gobierno del PP (Rajoy tomó posesión el 21 de diciembre de 2011, y durante el primer semestre de 2012 permanecieron en sus cargos el presidente de RTVE y el director de informativos de la última etapa del Gobierno de Zapatero, hasta que fueron sustituidos el 28 y el 29 de junio de 2012, respectivamente); y 2013 es el segundo año de mandato de Rajoy, una etapa en la que RTVE tiene un nuevo presidente que ha sido propuesto sin consenso porque el Gobierno de PP modificó la norma para que no fuese necesario el apoyo de los dos tercios del Congreso para el nombramiento.

Trabajamos con una muestra que comprende tres años de etapas diferentes, y de cada año seleccionamos 12 informativos mediante el método de la semana compuesta. Analizamos los telediarios de la primera edición, que se emite a las 15 horas, porque es la que ofrece mayor actualidad. Y descartamos los informativos del fin de semana porque, al no haber actividad parlamentaria, disminuye considerablemente la infor- 
mación política de carácter nacional, que es el elemento que más nos interesa como objeto de estudio.

\section{Objetivos e hipótesis}

El objetivo de esta investigación es determinar su hubo cambios en la línea editorial y en el organigrama de RTVE a partir de la toma de posesión del Gobierno del Partido Popular en diciembre de 2011 y del nombramiento de un nuevo presidente y de un nuevo director de informativos de la cadena pública en junio de 2012, con respecto a los criterios que se aplicaban en 2008 cuando gobernaba el PSOE. En el caso de que existan novedades, nos interesa conocer en qué consisten y cómo se reflejan los criterios informativos de cada etapa en la selección temática, en el enfoque y en el tratamiento de los contenidos. Luego también comprobaremos si hay cambios durante el periodo señalado en las televisiones privadas, comparando los informativos seleccionados de TVE con los de las mismas fechas de Antena3, de Cuatro, de Telecinco y de La Sexta.

Partimos de la hipótesis de que el consenso político sobre la televisión pública favorece el rigor, la transparencia y el pluralismo informativo, y disminuye la manipulación y la censura.

\section{Metodología}

De acuerdo con los objetivos de esta investigación, realizamos un análisis cuantitativo y cualitativo, y también comparativo y longitudinal. Esta metodología mixta permite trabajar conjuntamente con todos los elementos que forman parte de las diferentes dimensiones del relato informativo, comparar y contrastar los datos de cada nivel, conocer la evolución de los telediarios durante las tres etapas objeto de estudio y ver si hubo cambios significativos en algún momento del periodo seleccionado.

Existen varios factores que pueden afectar a una noticia: a) un enfoque tendencioso de los hechos para fabricar una versión favorable o crítica, según convenga; b) la estratificación, otorgándole un lugar más o menos preferente en la escaleta para aumentar o reducir artificialmente su importancia informativa; y c) el tiempo que se le adjudica, asignándole una duración inadecuada que no se corresponde con la relevancia de los hechos, para que destaque de una forma extraordinaria o para minimizar su trascendencia y su impacto.

En primer lugar realizamos un análisis cuantitativo para ver si las informaciones reúnen las exigencias de pluralismo formal (Humanes et al., 2013: web) y comprobamos si en el relato están incluidos todos los puntos de vista necesarios para la comprensión de la noticia y si los actores tienen una presencia proporcional al grado de protagonismo que les corresponde (González, 1989: web). Luego continuamos con un análisis cualitativo para conocer las particularidades del enfoque y del tratamiento informativo de los hechos. Pero, además del tratamiento y del enfoque, también analizamos la estratificación para ver cómo están organizados los contenidos y valorar el orden de emisión establecido para cada tema. Aquí comparamos las características de las noticias que integran el telediario con el lugar que ocupan en la escaleta, para determinar si hay una correspondencia lógica entre la relevancia del hecho y su ubica- 
ción (González, 1989: web). Se trata de ver si la posición se debe a criterios de raccord, de interés informativo o si existe una ubicación forzada para destacar o relegar algún acontecimiento.

Finalmente, comparamos los resultados de las fases anteriores para ver si existe coherencia entre el tratamiento formal de las noticias, el enfoque, el tratamiento informativo y la estratificación, o si existen diferencias que evidencian la aplicación de criterios distintos en cada caso. Prestamos una atención especial a la información política e institucional porque consideramos que es la que está sujeta a más presiones y en la que mejor se reflejan los posibles controles (Ortega, 2011).

Para conocer los protocolos que se siguen en los informativos de TVE, la investigación se completa con una entrevista a diez periodistas de la cadena pública, dos de cada una de las cinco áreas que integran la redacción, que han trabajado en los telediarios durante todo el periodo objeto de estudio y pueden acreditar si ha habido cambios significativos en alguna de las etapas analizadas. Y, en caso afirmativo, nos interesa saber en qué consisten esos cambios, cómo se han justificado ante la redacción, cómo repercuten en los procesos de producción de la información, en qué grado afectan a las garantías de independencia de los periodistas y cómo influyen en el enfoque y en el tratamiento de las noticias.

\section{Análisis de los datos y resultados}

Los resultados de las entrevistas realizadas a los redactores indican que el organigrama básico de los informativos de TVE, desde el punto de vista formal, es idéntico en las tres etapas y tampoco se advierten diferencias sustanciales en los esquemas de funcionamiento establecidos en todo el periodo estudiado. Las respuestas confirman que permanecen las estructuras, que se trabaja con recursos similares y con los mismos protocolos.

Los entrevistados confirman que la configuración de la redacción de informativos de TVE se mantiene invariable en las tres etapas, respetando las competencias temáticas atribuidas tradicionalmente a las áreas de especialización clásicas (Esteve Ramírez y Fernández del Moral, 1999). Los redactores también reconocen que los métodos de trabajo que forman parte de las rutinas productivas de la cadena permanecen y son ajenos a otros cambios más estratégicos, pensados para ejercer puntualmente el control de los contenidos a través del enfoque o del tratamiento informativo.

El 70\% dice que muchos controles de la información se realizan con procedimientos sofisticados, aprovechando la versatilidad de las estructuras consolidadas, utilizándolas de forma inadecuada e interesada y atribuyéndoles funciones y competencias circunstanciales que no son las que les corresponden habitualmente de forma natural y lógica. En este sentido, el $91 \%$ coincide en que la estructura del organigrama de los telediarios es operativa y aseguran que dispone de todos los recursos necesarios para ofrecer una información plural y de calidad, pero, al mismo tiempo, el 74\% mantiene que el esquema vigente es vulnerable y favorece el control de los contenidos porque no existen unos mecanismos que garanticen totalmente la independencia de los periodistas e impidan posibles extralimitaciones en sus funciones del director de informativos o de los jefes de área. 
E1 63\% se muestra contrario a los cambios sistemáticos en los puestos de responsabilidad cada vez que se incorpora un nuevo director de informativos, pero el $75 \%$ admite que en la redacción de TVE ya se asumen los ceses como algo natural, aunque no estén justificados profesionalmente. El responsable de informativos de la primera etapa analizada (2008), Fran Llorente, cambió todos los jefes de área que habían sido nombrados por su antecesor, luego mantuvo el mismo equipo durante el tiempo que continuó dirigiendo los telediarios en la segunda etapa, hasta finales de junio de 2012. Y el siguiente director de informativos, Julio Somoano, cesó el 80\% de los jefes de área que habían trabajado con Fran Llorente.

E1 79\% de los redactores afirma que el perfil profesional no es el único factor que se valora en el nombramiento y en la destitución de los jefes de área. Reconocen que la formación no siempre es un mérito suficiente para ascender y que las razones políticas e ideológicas con frecuencia son determinantes en el relevo de cargos directivos para garantizar un control de los contenidos permanente y exhaustivo. Sólo el $21 \%$ mantiene que los nombramientos están basados en criterios profesionales y que se rigen por méritos objetivos que avalan el perfil del candidato como idóneo para la nueva responsabilidad por su especialización. En este sentido, el $62 \%$ de los redactores asegura que muchas veces los ceses de los jefes de área se realizan sin explicaciones convincentes, argumentando que el director de informativos quiere trabajar con gente de confianza, una justificación que el $45 \%$ considera una excusa formal para enmascarar cambios que no se basan en criterios técnicos.

El 87\% de los redactores sostiene que los jefes de área son claves en el control de la información porque son los que supervisan directamente todas las noticias y los que deciden qué se controla y cómo se controla, qué temas se emiten, cuáles se descartan e incluso desde qué perspectiva se enfocan, y la selección temática y la orientación posterior de los hechos determinan el grado de pluralismo y de objetividad de los telediarios (Humanes et al., 2013: web). Además, el 81\% de los entrevistados afirma que los responsables de área alertan al director de informativos si consideran que una noticia requiere una atención especial y se aseguran de que la redacción final de determinados temas se ajuste a la línea editorial de la cadena.

E1 83\% reconoce que no todas las noticias pasan las mismas supervisiones y que el grado de control depende de muchas circunstancias. Así, confirman que determinados temas están sometidos a una vigilancia permanente, especialmente todo lo relacionado con la Casa Real y los asuntos más polémicos que forman parte de la acción de Gobierno, pero el $71 \%$ añade que, para evitar situaciones incómodas y asegurarse una versión acorde con la línea editorial establecida, las noticias más sensibles, habitualmente, son asignadas a redactores de confianza de la dirección de informativos porque conocen las consignas y, además, asumen todas las indicaciones sin plantear problemas.

Los resultados sobre el grado de independencia con el que aseguran haber desarrollado su actividad profesional los redactores de TVE en cada momento son muy diferentes. En 2008 el 15\% de los entrevistados dice que ha trabajado con un margen limitado, el 45\% lo califica como satisfactorio y el 40\% lo considera óptimo. En 2012 el 9\% mantiene que ha tenido limitaciones impuestas, el 30\% califica la experiencia como satisfactoria y el 61\% la valora como óptima. En cambio, en 2013 hay descon- 
tento en la mayoría de la redacción. El 56\% reconoce que ha tenido impedimentos puntuales para realizar su trabajo con libertad, el 33\% califica la independencia profesional como satisfactoria y sólo el $11 \%$ reconoce que ha trabajado en condiciones óptimas.

Pero para el $68 \%$ el grado de libertad con que se trabaja no es permanente, depende mucho de los temas, y también de las circunstancias, porque, según matizan, un tema puede ser conflictivo en un contexto determinado y luego estar liberado de presiones cuando pierde vigencia o deja de ser polémico. Uno de los ejemplos que mencionan es el paro, y aclaran que se trata de forma diferente durante la crisis que en época de bonanza.

\subsection{El pluralismo formal en las noticias de TVE}

Ahora nos centramos exclusivamente en el tratamiento formal de las noticias, sin entrar en valoraciones cualitativas, ya que este aspecto se analiza en un apartado posterior.

Además de los criterios técnicos que se aplican para editar con coherencia una noticia en televisión, existen otros requisitos para garantizar que en el relato audiovisual tengan presencia todos los puntos de vista necesarios (González, 1989: web). Por eso, para conocer el grado de pluralismo formal (Humanes et al., 2013: web), nos interesa saber si en las noticias analizadas están incluidos, desde un parámetro cuantitativo, todos los elementos esenciales para que el acontecimiento conserve su identidad inicial y la versión sea correcta, de acuerdo con las características y con la naturaleza de los hechos (González, 1989: web).

Los resultados obtenidos demuestran que las noticias de TVE cumplen los requisitos técnicos y formales propios de una información en un porcentaje muy alto, según los criterios de calidad utilizados como referencia en esta investigación (Gómez Mompart et al., 2013). Basándonos en esos parámetros, el tratamiento formal del $95 \%$ de las noticias analizadas en 2008 es correcto. En el 97\% de las informaciones de 2012 todos los elementos y puntos de vista tienen una presencia cuantitativa en el relato proporcional a su grado de importancia. Y los resultados observados en 2013 son muy similares a los anteriores. Aquí los tratamientos formales correctos alcanzan el 93\% de las noticias.

\subsection{El pluralismo formal en la información política}

Los redactores entrevistados han confirmado que los criterios para asignar el reparto de los tiempos de las noticias relacionadas con los partidos políticos se mantienen invariables, al margen de los cambios que se producen periódicamente en la dirección de informativos. TVE aplica una fórmula consensuada por los dos grandes partidos, PSOE y PP, creada inicialmente para regular y distribuir los espacios de propaganda en televisión durante las campañas electorales, que consiste en asignar a cada partido un tiempo proporcional a su representación parlamentaria (Bustamante, 2013). Este esquema terminó trasladándose a los telediarios y se convirtió en uno de los criterios principales por los que se rigen y diseñan los minutados, consolidándose como una solución para dar voz diariamente a los partidos en la televisión pública de forma automática y garantizar así el pluralismo formal de la información política, una solución 
que a veces resulta incompatible con los criterios periodísticos y con la amplitud y forma de tratamiento que requiere la trascendencia de los hechos. Pero el grado de pluralismo formal no se corresponde siempre con los índices de neutralidad registrados en los enfoques y en los contenidos, como veremos luego al exponer los datos de los análisis de estos aspectos.

Para comprobar si existe una correspondencia entre los tiempos de las noticias referidas a los partidos políticos españoles y la representación parlamentaria de esas formaciones comparamos el porcentaje que suman los escaños de cada partido, con respecto al total de diputados que componen el Parlamento, con el porcentaje que representa el tiempo de la información de cada partido emitida en los telediarios analizados, con respecto al total del tiempo que suman las noticias de información política nacional registrada en eses mismos informativos.

Contabilizamos los tiempos asignados a cada partido en cada telediario y no el número de noticias porque consideramos que es más representativo, ya que durante el análisis hemos detectado que, en algunas ocasiones, una misma noticia consta de varias entregas informativas. Por ejemplo, cuando un acontecimiento se está desarrollando mientras se emite el telediario, es habitual que se produzcan varias conexiones para conocer en directo la evolución de los hechos. Y esas conexiones no contabilizan como noticias diferentes porque corresponden al mismo acontecimiento.

$\mathrm{El}$ análisis de los telediarios demuestra que existe un paralelismo permanente entre la composición del arco parlamentario español y el grado de presencia formal de los diferentes partidos en los informativos de la televisión pública, una constante que se mantiene invariable en las tres etapas analizadas, al margen de interés periodístico y del enfoque de cada noticia. De hecho, en el $21 \%$ de los casos la duración de las noticias relacionadas con los partidos políticos españoles no es proporcional a su interés informativo, si tomamos como referencia los criterios de noticiabilidad establecidos por González (1989: web), que son los que utilizamos como modelo de trabajo en esta investigación. Y siguiendo esos criterios, en el $17 \%$ de los casos el orden de emisión asignado a los temas en el telediario tampoco se corresponde con la ubicación que deberían tener en la escaleta atendiendo a la relevancia de los hechos. $\mathrm{Al}$ aplicar la fórmula mencionada antes, automáticamente las informaciones protagonizadas por los partidos mayoritarios son más amplias y esa solución condiciona la estratificación de los contenidos. Así las noticias relacionadas con el PSOE y con el PP tienen un tratamiento cuantitativo privilegiado.

Los siguientes datos demuestran que al realizar una comparativa entre el número de escaños que posee cada partido, que son los que configuran su representación parlamentaria durante la legislatura, y el tiempo que se le dedica a esa formación política en los informativos de TVE existe una correspondencia proporcional permanente entre ambos factores. Exponemos sólo los tiempos registrados en los telediarios sobre las informaciones referidas al PSOE, al PP y a CiU, los tres partidos con mayor representación parlamentaria en las dos legislaturas que pertenecen al periodo analizado, porque consideramos que estos datos son suficientes para reflejar las consecuencias de la fórmula aplicada por la cadena pública a la hora de minutar las noticias de contenido político. 
En 2008 los escaños obtenidos por el PSOE representan el 48\% del arco parlamentario y las informaciones referidas a este partido que se emiten en los telediarios analizados suman el $46 \%$, con respecto al total del tiempo que ocupa la información de los partidos políticos españoles. Los escaños del PP representan el $44 \%$ y el tiempo de esta formación alcanza el 41\%, y los escaños de CiU representan el 3\% y el tiempo que se le asigna contabiliza el 5\%.

Los datos de 2012 evidencian que ha variado la correlación de fuerzas políticas con respecto a 2008 y que se ha producido un cambio de Gobierno. En esta legislatura el PP obtuvo el 53\% de los escaños y la información que protagonizada suma el $51 \%$. Este dato refleja que el nuevo Gobierno dispone de mayoría absoluta porque el tiempo asignado en los telediarios de 2012 al PP es superior al que registraba el PSOE en 2008. Ahora los escaños del PSOE suman el $31 \%$ y este dato explica que tenga una presencia menor en los telediarios, el $27 \%$, de acuerdo con la nueva situación. CiU ha aumentado su representación, consigue el 4,6\% de los escaños y esta circunstancia se refleja con una presencia en los informativos del 7\%.

La composición parlamentaria de 2013, año en el que sigue gobernando el PP, no varía y los datos de los tiempos de las noticias protagonizadas por los tres principales partidos son similares a los registrados en 2012: las informaciones referidas al PP suman el 54\%, las relacionadas con el PSOE el 30\% y las de CiU el 8\%. Estos resultados demuestran que, aunque ha cambiado el director de informativos, se mantienen los mismos criterios para la asignación de tiempos a la información política y los partidos conservan un grado de presencia estable en los telediarios, según su representación parlamentaria, al margen del interés que tengan las noticias que generan.

Además del control de los tiempos que garantiza la fórmula implantada con el consenso del PSOE y del PP, al analizar la información política e institucional referida a España se detecta que en los telediarios de las tres etapas permanecen los mismos protagonistas en la mayoría de los casos, una continuidad favorecida por la estructura, por el funcionamiento y por el sistema de representación que tienen establecido los partidos para canalizar sus mensajes. La fórmula vigente evita, reduce o dificulta la presencia en los informativos de voces críticas y fomenta la estandarización del discurso político y el protagonismo permanente de los mismos actores. Las relaciones con los medios están reguladas y las opiniones que representan corrientes alternativas no disponen de cauces para hacerse oír porque cada partido tiene unos representantes que son las únicas voces autorizadas (Berrocal, 2000). Los datos demuestran que predomina el discurso oficial y que hay una escasa renovación en los órganos de representación. E1 83\% de los políticos que aparecen en los telediarios como voces oficiales de sus respectivos partidos son los mismos en 2008, en 2012 y en 2013, y su grado de protagonismo, cualitativo y cuantitativo, varía en cada momento como consecuencia natural del cambio de Gobierno, que implica también un relevo de cargos oficiales en el ámbito político e institucional y la asunción de responsabilidades por parte de otro partido que estaba en la oposición.

En este sentido, el 78\% de los redactores entrevistados reconocen que en TVE, a la hora de consultar fuentes, hay una tendencia a acudir a los órganos de representación establecidos por los propios partidos, que son los que facilitan la versión oficial 
sobre sus propuestas, estrategias y acciones. Además, así se evitan protestas de los miembros del Consejo de Administración de RTVE, que son propuestos por las formaciones políticas y vigilan que su partido tenga una representación adecuada y que predomine o que trascienda sólo el discurso oficial, que es el que se corresponde con las tesis del líder (Casero Ripollés, 2008: web).

El $81 \%$ de las intervenciones de políticos registradas en las noticias analizadas en 2008 corresponden a miembros que forman parte de los órganos de dirección de los partidos, un porcentaje que se mantiene alto en 2012 con el $77 \%$ y en 2013 con el $85 \%$. En cambio, la presencia de voces alternativas, críticas o disidentes representa el $19 \%$ en 2008 , el $23 \%$ en 2012 y el $15 \%$ en 2013 .

En 2008 en el $17 \%$ de las noticias protagonizadas por el PSOE se mezcla la información política con la institucional y se hace una referencia conjunta a las propuestas del partido mayoritario y a la acción de Gobierno, sin separar los roles y las responsabilidades de cada uno, algo que favorece al partido gobernante. Esta misma característica se advierte en el $10 \%$ de las noticias de 2012 y en el $21 \%$ de las de 2013 , pero ahora en acontecimientos protagonizados por el PP.

\subsection{Los predominios temáticos de cada etapa}

La mayoría de las noticias pueden ubicarse en diferentes secciones según qué criterio se aplique en el enfoque, por eso aquí utilizamos como referente para catalogar un tema el elemento al que el redactor que elabora esa información le otorga el rol de sujeto principal y lo destaca sobre los demás detalles que integran el acontecimiento. Así situamos cada noticia en su área correspondiente basándonos en un criterio homogéneo. Esta solución nos permite realizar un análisis riguroso y conocer qué temas predominan en cada etapa.

Al analizar los contenidos de los telediarios se comprueba que el grado de presencia de las diferentes temáticas ha ido variando según el contexto (Pestano Rodríguez, 2008: web). Esta evolución responde a las tendencias naturales marcadas por circunstancias que modifican e imponen las referencias informativas. Los intereses y los problemas de los ciudadanos cambian con el tiempo y los medios reflejan lo que pasa para dar una respuesta adecuada a la sociedad (Díaz Arias, 2008: web). Exponemos los datos de las noticias de economía, de política y de sociedad, que son las que mejor representan la evolución temática del periodo analizado.

En 2008 hay un equilibrio temático que demuestra que no existen preocupaciones especiales por ninguna cuestión: las noticias de sociedad ocupan el primer lugar (32\%), pero también tienen una presencia destacada las de economía (29\%) y las de política (24\%). El año 2012 coincide con la crisis económica, el paro aumenta considerablemente y los telediarios reflejan los problemas laborales y las consecuencias del desempleo. Ahora las informaciones de carácter económico ocupan el primer lugar (41\%) y predominan sobre las de contenido político $(27 \%)$ y sobre las de sociedad (21\%). En 2013 la situación del país es crítica porque la crisis se agrava, el paro sigue creciendo y España está en riesgo de ser rescatada, y esta circunstancia influye en el peso informativo que adquiere el área que tiene competencias sobre el tema que preocupa a muchos ciudadanos españoles. Las noticias de economía representan más de 
la mitad de todas las analizadas este año y alcanzan el $53 \%$, frente al $22 \%$ que suman las de política y al $18 \%$ que corresponde a las de sociedad.

La selección temática es un indicador que refleja las tendencias, pero el análisis de los enfoques permite conocer las características de la línea editorial establecida, que es la que marca la política informativa que se aplica en cada etapa.

\subsection{La forma y el fondo: la correspondencia entre el tratamiento formal y el en- foque de las noticias}

El alto grado de pluralismo formal que se registra en las noticias de TVE en las tres etapas objeto de estudio no se corresponde siempre con un enfoque y con un tratamiento riguroso de los hechos, algo que se comprueba al comparar los datos del análisis cuantitativo con los resultados obtenidos en el análisis cualitativo. La diferencia que existe entre el índice de objetividad y de neutralidad de ambas dimensiones demuestra que se aplican criterios distintos en el tratamiento formal, en el tratamiento informativo y en el enfoque, especialmente en los temas de contenido político y más concretamente en las informaciones referidas al Gobierno y a la oposición, que es donde se acentúan los contrastes entre la forma y el fondo.

En el $9 \%$ de las noticias de 2008 el rigor del pluralismo formal no se corresponde con un tratamiento de las mismas características en el planteamiento del enfoque ni en el desarrollo del relato, que no se ajustan a los parámetros de calidad aplicados en esta investigación (Gómez Mompart et al., 2013). Y de los casos en los que se detectan carencias, el 52\% son temas relacionados con la información política e institucional. En las noticias analizadas en 2012 también se advierte disparidad de criterio entre la dimensión cuantitativa y cualitativa del relato en el $11 \%$, y el $47 \%$ de las diferencias se localizan en noticias de las mismas características que en los ejemplos de 2008. $\mathrm{Y}$ en 2013 en un $23 \%$ de las informaciones tampoco existe una correspondencia coherente entre la forma y el fondo, y el $61 \%$ de los enfoques o tratamientos tendenciosos también pertenecen a la información política e institucional.

Es importante destacar aquí que los redactores entrevistados confirman que a partir del mes de junio de 2012, fecha en la que toma posesión el nuevo director de informativos de TVE, la mayoría de los periodistas de la cadena pública que hasta ese momento se encargaban de la cobertura de la información parlamentaria son sustituidos por otros redactores.

\subsection{La línea editorial de TVE}

Para comprobar si existen cambios en la línea editorial de los informativos de TVE en alguna de las tres etapas objeto de estudio con respecto a las demás, pedimos a los redactores entrevistados que seleccionaran cinco temas sobre los que el PSOE y el PP mantienen criterios diferentes o posturas antagónicas y que tengan constancia de que han sido sometidos en algún momento o de forma permanente a controles rigurosos en la redacción de la cadena pública, y los contenidos más señalados son los siguientes, por este orden: la crisis económica, el paro, la sanidad, la educación y las reivindicaciones autonómicas. Todos estos temas aparecen reiteradamente como protagonistas de la información política e institucional mencionada antes, y son re- 
presentativos de la actualidad porque se trata de cuestiones transversales y de interés general que han sido referencias informativas y que han tenido una vigencia destacada y permanente desde 2008 a 2013. Son temas significativos porque tienen repercusiones en el ámbito político, económico y social y han condicionado la acción de Gobierno y las reacciones de la oposición. Además, configuran el discurso ideológico y definen las principales líneas de actuación del PSOE y del PP, que son los dos partidos que han gobernado durante el periodo analizado.

De los cinco temas marcados por los redactores, para hacer un seguimiento de la línea editorial de TVE, seleccionamos las noticias relacionadas con la crisis económica, que es el punto sobre el que existe más consenso por parte de los entrevistados a la hora de señalar contenidos sobre los que se ha ejercido un control exhaustivo de la información por parte de la cadena pública. Al realizar un análisis del tratamiento de la crisis durante las tres etapas objeto de estudio es posible determinar de una forma más precisa el grado de control de la información que existe en cada momento y ver si varía el enfoque y la posición sobre un mismo tema o si permanecen los criterios ya establecidos en etapas anteriores. Y para tener una perspectiva más amplia, comparamos el enfoque de las noticias relacionadas con la crisis económica que han sido emitidas en TVE con el enfoque de las noticias de las mismas características localizadas en Antena 3, en Cuatro, en Telecinco y en La Sexta, para conocer los criterios informativos de cada cadena respecto al mismo tema y ver si hay diferencias entre las distintas televisiones (Segado Boj et al., 2013).

Como en una noticia hay voces diferentes, exponemos los tiempos que suman las versiones favorables y las críticas para conocer cuál es el discurso dominante.

En 2008 la crisis aún no afectaba a la sociedad española y esta circunstancia se refleja en el enfoque de las noticias de TVE. El discurso dominante sobre la economía del país es positivo y en el $74 \%$ del tiempo dedicado a informar sobre este tema en los telediarios se niega la existencia de problemas. Además, se asegura que España está preparada para afrontar la crisis y que no va a influir en la situación económica de forma dramática. Cuatro, Telecinco y La Sexta mantienen un discurso similar al de TVE, alineado con las tesis del Gobierno de Zapatero, basado en datos oficiales y, además, reforzado con declaraciones institucionales. Pero el enfoque positivo y tranquilizante de TVE sobre la economía contrasta con las versiones de Antena 3. En esta cadena, en el $61 \%$ del tiempo que dedica a informar sobre la situación del país ya se advierte de la gravedad del tema, se alerta sobre las posibles consecuencias de una recesión y se critica la pasividad del Ejecutivo ante la situación que se avecina, basándose en las opiniones de voces críticas con el Gobierno, principalmente miembros de la oposición y expertos que no comparten la política económica de Zapatero.

En 2012 TVE ya tiene otro discurso informativo. Ahora no niega la existencia de la crisis y la trata como una realidad que afecta a todos los sectores. Pero, para analizar los telediarios con rigor, este año hay que diferenciar dos momentos. En el primer semestre permanece Fran Llorente como director de informativos y la cadena pública mantiene un enfoque tranquilizador sobre la economía. La crisis se presenta como algo inevitable y en el 78\% del tiempo que se dedica a informar sobre el tema se relativizan los datos negativos referentes a la situación del país, se minimizan las con- 
secuencias del posible impacto social del paro y se justifican las medidas adoptadas por el Gobierno. Aquí todavía coincide el discurso dominante de TVE con la política informativa de Cuatro, de Telecinco y de La Sexta, y permanecen las diferencias con Antena 3.

A finales de junio de 2012 toma posesión Julio Somoano como nuevo director de informativos y se produce un cambio en la línea editorial de TVE. A partir de este momento las noticias se enfocan desde una perspectiva diferente. En el 75\% del tiempo que se dedica a informar sobre la crisis se reconoce abiertamente su impacto sobre la sociedad en general, pero se responsabiliza al Ejecutivo anterior de la situación. Además, en la mayoría de las noticias emitidas por la cadena pública se justifican las medidas y los recortes anunciados por el Gobierno, que afectan al ámbito económico y social, y se presentan como algo necesario e inevitable. Desde que dirige los informativos Julio Somoano ya no se mantienen las afinidades entre las mismas cadenas. Ahora TVE coincide mayoritariamente en los enfoques con Antena 3 y se aleja de los planteamientos de Cuatro, de Telecinco y de La Sexta.

Los análisis de los telediarios demuestran que en 2013 TVE mantiene la misma línea editorial que establece en junio de 2012. En el 82\% del tiempo que se dedica a informar sobre la crisis se continúa presentando la situación económica que atraviesa el país como un problema heredado del Gobierno anterior. En 2013 el discurso dominante de TVE también coincide con el de Antena 3 y se diferencia del de Cuatro, Telecinco y La Sexta, que culpan al Gobierno de Rajoy de la evolución de la crisis y critican las medidas de ajuste impuestas por Europa, mientras que la cadena pública presenta una imagen positiva del Gobierno, construida con noticias que le son favorables.

La comparativa anterior refleja un cambio en el enfoque informativo de la crisis por parte de TVE con respecto al criterio que mantenía antes. Esto evidencia una alineación permanente de la cadena pública con el discurso del Gobierno, algo que se traduce en un control inadecuado de los contenidos y en una politización de la información.

\section{Conclusiones}

Los resultados de la investigación confirman la hipótesis principal. Los mayores índices de pluralismo y de neutralidad se localizan en los telediarios de 2008 y en los del primer semestre de 2012, mientras que los niveles más elevados de enfoques tendenciosos y de politización de la información se registran en el segundo semestre de 2012 y en 2013, cuando RTVE vuelve a estar dirigida por un presidente nombrado de forma unilateral por el Gobierno, sin consenso con los demás partidos. En la información política es donde se detectan los grados más bajos de objetividad, y esta particularidad es una constante en todo el periodo analizado.

Existe coincidencia entre el enfoque que se hace en los telediarios de la mayoría de las noticias relacionadas con la gestión y con la acción de Gobierno y las posiciones y las tesis que mantienen el Ejecutivo y el partido que lo sustenta, lo que demuestra que hay una alineación permanente de TVE con los criterios oficiales. El grado de coincidencia de ambos posicionamientos también es mayor en el segundo semestre de 2012 y en 2013. 
Al comparar los tratamientos informativos de las tres etapas objeto de estudio se detecta que los mismos temas tienen enfoques diferentes, e incluso contradictorios, según la política informativa que se aplique en cada momento. Los resultados de los análisis de contenido evidencian un cambio de criterio y una nueva línea editorial desde que se nombra un presidente de RTVE sin consenso y un nuevo director de informativos. Estos cambios impiden mantener un criterio único y consolidarlo como referencia, para garantizar un tratamiento homogéneo de los contenidos, independientemente de circunstancias o de intereses puntuales. Además, las contradicciones desconciertan a la audiencia y perjudican la credibilidad informativa de la cadena pública.

El grado de pluralismo formal es similar en los telediarios de 2008, de 2012 y de 2013, y no se registran diferencias significativas referentes a este aspecto en ninguna sección. Pero pluralismo formal no garantiza un pluralismo real y no se corresponde con los índices de objetividad y de neutralidad que se desprenden de los análisis de contenido. Los resultados reflejan que en los enfoques y en los tratamientos informativos de las noticias no existe siempre una correspondencia entre la dimensión cuantitativa y cualitativa del relato.

El criterio que se aplica para mantener la presencia diaria de todos los partidos en TVE implica una limitación que afecta a la pluralidad entendida en el sentido más amplio de representación informativa de la sociedad, y no sólo como pluralidad política. Los compromisos permanentes que existen con los partidos ocupan buena parte del espacio y del tiempo de los telediarios e impiden la presencia de otros temas.

La estabilidad que se aprecia en el organigrama, en los protocolos y en los criterios por los que se rige la catalogación, distribución y asignación de la información a las distintas áreas que integran la redacción de los telediarios demuestra que no existen diferencias estructurales significativas entre las tres etapas objeto de estudio. Esto corrobora que los modos y los sistemas de producción informativa están consolidados y son ajenos a los cambios puntuales o periódicos en la línea editorial y en el organigrama directivo de TVE.

\section{Referencias bibliográficas}

BERROCAL, Salomé (Coord., 2000): Comunicación politica en televisión y nuevos medios. Barcelona, Ariel Comunicación.

BUSTAMANTE, Enrique (2013): Historia de la Radio y la Televisión en España. Una asignatura pendiente de la democracia. Barcelona, Gedisa.

CAMPOS FREIRE, Francisco (2013): "El futuro de la TV europea es híbrido, convergente y cada vez menos público". Revista Latina de Comunicación Social: http://www.revistalatinacs.org/ 068/paper /970_Santiago/04_Campos.html [Consulta: 10 de marzo de 2014]

CASERO RIPOLLÉS, Andreu (2008): "Modelos de relación entre periodistas y políticos: la perspectiva de la negociación constante". Estudios sobre el Mensaje Periodístico, vol. 14, pp. 11-128. Madrid, Servicio de Publicaciones de la Universidad Complutense: http://revistas.ucm.es/index.php/ESMP/article/view/ESMP0808110111A [Consulta: 7 de febrero de 2014] 
CASTILLO ESPARCIA, Antonio (2012): "Los medios de comunicación como actores sociales y políticos. Poder, medios de comunicación y sociedad", en Razón y Palabra: www.razonypalabra.org.mx/N/N75/monotematico_75/12_Castillo M75.pdf [Consulta: 7 de marzo de 2014]

DE MATEO, Rosario y BERGÉS, Laura (2008): Los retos de las televisiones públicas. Financiación, servicio público y libre mercado. Sevilla, Comunicación Social.

DÍAZ ARIAS, Rafael (2006): Periodismo en televisión: entre el espectáculo y el testimonio de la realidad. Barcelona, Bosch.

DÍAZ ARIAS, Rafael (2008): "La representación del mundo en los informativos de televisión". Estudios sobre el Mensaje Periodístico, vol. 14, pp. 363-384. Madrid, Servicio de Publicaciones de la Universidad Complutense: http://pendientedemigracion.ucm.es/info/emp/Numer_14/Sum/4-05.pdf [Consulta: 8 de febrero de 2014]

ESTEVE RAMÍREZ, Francisco y FERNÁNDEZ DEL MORAL, Javier (1999): Áreas de especialización periodística. Madrid, Fragua.

GARCÍA AVILÉS, José Alberto (2007): "El infoentretenimiento en los informativos líderes de audiencia en la Unión Europea”. Anàlisi: http://ddd.uab.cat/pub/analisi/02112175n35p47.pdf [Consulta: 24 de enero de 2014]

GÓMEZ MOMPART, Josep Lluís; GUTIÉRREZ LOZANO, Juan; y PALAU SAMPIO, Dolors (eds. 2013): La calidad periodística. Teorías, investigaciones y sugerencias profesionales. Valencia, Servei de Publicacións Universitat de Valencia.

GONZÁLEZ, Norberto (1989): "Hechos y valores en la narración periodística informativa". Comunicación y Sociedad: http://www.unav.es/fcom/comunicacionysociedad/es/articulo.php?art_id=295 [Consulta: 10 de enero de 2014]

HUMANES, María Luisa (2001): "El encuadre mediático de la realidad social. Un análisis de los contenidos informativos en televisión". Zer: http://www.ehu.es/zer /es/hemeroteca/articulo/el-encuadre-mediatico-de-la-realidad-social-un-analisisde-los-contenidos-informativos-en-television1/153 [Consulta: 3 de marzo de 2014]

HUMANES, María Luisa; MONTERO SÁNCHEZ, María Dolores; MOLINA DE DIOS, Ramón; y LÓPEZ-BERINI, Alfredo (2013): "Pluralismo y paralelismo político en la información televisiva en España". Revista Latina de Comunicación Social: http://plataformarevistascomunicacion.org/2013/10/pluralismo-y-paralelismo-politico-en-la-informacion-televisiva-en-espana/ [Consulta: 7 de enero de 2014]

LEÓN, Bienvenido (coord., 2010): Informativos para la televisión del espectáculo. Sevilla y Zamora, Comunicación Social.

ORTEGA, Félix (2011): La política mediatizada. Madrid, Alianza.

ORTELLS BADENES, Sara (2009): "La mercantilización de la información: la nueva era informativa en televisión". Revista Latina de Comunicación Social: $\mathrm{http} / / / \mathrm{www} . r e v i s t a l a t i n a c s . o r g / 09 / a r t / 28$ 827_46_ULEPICC_07/Sara_Ortells.html [fecha de consulta: 21 de enero de 2014] 
PALACIO, Manuel (2001): Historia de la televisión en España. Barcelona, Gedisa.

PALACIO, Manuel (2012): La televisión durante la transición española. Madrid, Cátedra.

PÉREZ ORNIA, José Ramón (1989): Peculiaridades de una televisión gubernamental. Barcelona, Ariel.

PESTANO RODRÍGUEZ, José (2008): “Tendencias actuales en la estructura y contenidos de los informativos de televisión". Revista Latina de Comunicación Social: http://www.revistalatinacs.org/08/38_795_60_TV/Jose_Pestano_Rodriguez .html [fecha de consulta: 9 de enero de 2014]

RETIS, Jéssica; LAMUEDRA, María; y GARCÍA MATILLA, Agustín (2010): Los informativos diarios en BBC y TVE. Madrid, Ediciones de la Torre.

SEGADO BOJ, Francisco; FERNÁNDEZ GÓMEZ, Erika; GIL GASCÓN, Fátima; y MARTÍN QUEVEDO, Juan (2013): La crisis económica en los informativos de televisión. Logroño, Universidad Internacional de La Rioja.

SOLER CAMPILLO, María (2005): "El informe del "Comité de sabios": bases para una televisión de calidad". Comunicar: http://www.revistacomunicar.com /index.php? contenido $=$ detalles\&numero $=25 \&$ articulo $=25-2005-104$ [Consulta: 17 de marzo de 2014] 